\title{
Tumor size predicts prognosis of head and neck synovial cell sarcoma
}

\author{
ALIMUJIANG WUSHOU ${ }^{1 *}$ and XIN-CHAO MIAO ${ }^{2 *}$ \\ ${ }^{1}$ Cancer Research Institute, Fudan University Shanghai Cancer Center; Department of Oncology, Shanghai Medical College, \\ Fudan University, Shanghai 200032; ${ }^{2}$ Department of Oral and Maxillofacial Surgery, Ninth People's Hospital, \\ Shanghai Jiao Tong University School of Medicine, Shanghai 200011, P.R. China
}

Received March 22, 2014; Accepted September 26, 2014

DOI: $10.3892 / \mathrm{ol} .2014 .2634$

\begin{abstract}
Head and neck synoviosarcoma (HNSS) is uncommon. To the best of our knowledge, the specific clinicopathological characteristics, treatment outcome and prognostic factors of HNSS were uninvestigated at the time of writing, so a meta-analysis was performed. An online data collection was carried out using PubMed and Google Scholar. Studies that reported primary HNSS and the treatment, follow-up time and outcome were chosen for the present study. In total, 93 cases from 26 studies were included for analysis. The study sample consisted of 55 males and 38 females and the median age was 32.1 years (range, 4-76 years). The median follow-up period was 62.1 months (range, 1-373 months). The tumor size was correlated with local recurrence and metastasis of HNSS, as well as with mortality $(\mathrm{P}=0.001, \mathrm{P}<0.0001$ and $\mathrm{P}<0.0001$, respectively). The three-year, five-year and 10 -year survival rates were $82.1,80.4$ and $78.2 \%$ for treatment with surgery alone, and $88.5,85.5$ and $82 \%$ for treatment with surgery plus radiotherapy, respectively. A significant tumor size-dependent difference was found between the overall survival (OS) rates $(\mathrm{P}<0.0001)$, as tumors that were $>5.0 \mathrm{~cm}$ in diameter were associated with a worse OS rate (hazard ratio, 6.460; 95\% confidence interval, 206-18.917; $\mathrm{P}=0.001)$. The tumor size was found to be an independent adverse prognostic factor for the OS of HNSS patients. In conclusion, surgical excision is a mainstream treatment of HNSS and post-operative adjuvant radiotherapy improves the OS rate of HNSS patients.
\end{abstract}

Correspondence to: Dr Alimujiang Wushou, Cancer Research Institute, Fudan University Shanghai Cancer Center, 270 Dong'an Road, Shanghai 200032, P.R. China

E-mail: alimjan.wushur@gmail.com

*Contributed equally

Key words: synoviosarcoma, head and neck, treatment, outcome, meta-analysis

\section{Introduction}

Synovial cell sarcoma, or synoviosarcoma, (SS) is a mesenchymal malignancy that is termed SS since its histological appearance is similar to that of the synovium. However, SS rarely exhibits a synovial structure and is considered to originate from pluripotent mesenchymal cells (1). The characteristic biphasic pattern of SS is due to the two morphologically distinct but histogenetically related cell types that compose the sarcoma. Depending on the relative prominence of the two cell populations and the degree of differentiation, these tumors form a continuous histopathological spectrum of biphasic, monophasic fibrous, monophasic epithelial and poorly differentiated (round-cell) types (2). Since SS can be slow-growing, appear to be benign on imaging studies, vary in size and cause pain resembling that associated with trauma, SS is the most commonly misdiagnosed soft tissue malignancy $(3,4)$. The diagnosis of SS is made on the basis of its relatively distinctive, yet markedly variable, histopathological appearance in conjunction with histochemical findings, immunohistochemistry, electron microscopy and cytogenetic analysis, which have proved valuable in confirming morphological diagnoses $(5,6)$.

$\mathrm{SS}$ is a distinct soft tissue sarcoma that tends to be located in the extremities (2). The lower extremities account for $70 \%$ of cases, whereas SS is uncommon in the head and neck region, with only $3 \%$ of SS tumors located there (7). Due to low clinical morbidity, non-specific symptoms and heterogeneous histopathological features, head and neck SS (HNSS) is often misdiagnosed (8). As a result, clinical diagnosis and treatment planning remain a challenge (9). To the best of our knowledge, there have been no controlled studies to define the optimal management protocol for HNSS, and the treatment methods reported include surgery, chemotherapy, radiotherapy and multiple treatment modalities, with variable results. In addition, no specific prognostic factors of HNSS have been reported to date. The aims of the present study were to review the clinicopathological characteristics of HNSS in head and neck patients, report and compare the treatment options, and identify the prognostic factors of mortality.

\section{Materials and methods}

Selection of studies. A systematic literature search was performed using PubMed and Google Scholar. The search 
strategy was based on the combination of text words: 'Synoviosarcoma OR synovial sarcoma OR synovial cell sarcoma', 'head and neck region', 'upper aerodigestive tract', 'oral and maxillofacial region', 'sinonasal region' and 'neck'. For the literature search in PubMed, no lower date limit was utilized and the upper date limit was October 31,2013. Despite the fact that no language restrictions were initially imposed, the full-text review and the final analysis were limited to studies published in English. The references of all the retrieved studies were searched for additional relevant studies to enlarge the scope of the literature search.

Eligible criteria. A study was included for analysis if it reported a human study and histologically confirmed primary HNSS, provided a clear description of any treatment, reported a definite follow-up time of more than month, and provided the treatment outcome. The study was excluded if it reported recurrent or metastatic HNSS, or synchronous or metachronous multiple cancers in other organs or diseases, and if the study was a case series providing a mean or medium follow-up time.

Data extraction. A data extraction sheet was developed. The data extracted for each patient consisted of the age, gender, tumor history, tumor presentation, tumor size, tumor extension, lymphadenopathy status, surgery type, surgical margins, presence of neck dissection, histological grade, adjuvant therapy provided, follow-up time and treatment outcome. Not all studies contained all these pieces of data; however, they were included in the present analysis if the treatment and outcome were provided. In certain cases, the patients had more than one treatment and, thus, only the final treatment received was included in the comparison of treatments.

Statistical analysis. The $\chi^{2}$ or Fisher's exact tests for categorical variables were used for two-group comparisons of the clinicopathological parameters. Differences in the numerical variables were assessed using Student's t-test or non-parametric Wilcoxon test. Significant variables identified by univariate analysis were then entered into binary logistic regression models to identify independent predictors of mortality. The odds ratio and $95 \%$ confidence interval (CI) were reported for the logistic regression model. For time-to-event analysis, Kaplan-Meier curves were plotted and the log-rank test was used. Analysis of the effect of prognostic factors on cause-specific survival was undertaken using Cox proportional-hazards regression. When $\mathrm{P}<0.05$, the difference was regarded as statistically significant. All the statistical tests were two-tailed and all the data were analyzed using SPSS 18.0 software for Windows (SPSS, Inc., Chicago, IL, USA).

\section{Results}

Patient demographics. In total, 93 cases from 26 studies met the eligibility criteria for inclusion in the present analysis (8,10-34). The details of the identification and selection of the studies are presented in Fig. 1. The 93 patients consisted of 55 male and 38 female patients, providing a male-to-female ratio of 1.44:1.

The median age at the time of diagnosis was 32.1 years (range, 4-76 years).
Table I. Baseline characteristics, tumor site distribution and treatment type of 93 patients with head and neck synoviosarcoma.

\begin{tabular}{lr}
\hline Feature & Value \\
\hline Age, years & \\
Median & 32.1 \\
Range & $4-76$ \\
Gender, $\mathrm{n}$ & \\
Male & 55 \\
Female & 38 \\
Site, n & \\
Upper aerodigestive tract & 47 \\
Neck & 25 \\
Skull base & 13 \\
Other & 8 \\
Treatment type, $\mathrm{n}$ & \\
S & 39 \\
S+R & 26 \\
S+R+C & 19 \\
S+C+R & 3 \\
Other & 6 \\
\end{tabular}

S, surgery; R, radiotherapy; C, chemotherapy.

Tumor location, treatment and follow-up. In total, 50.5\% of the tumors were located in the upper aerodigestive tract, $26.9 \%$ in the neck and $14.0 \%$ in the skull base. The treatment modalities consisted of surgery (41.9\%), surgery plus radiotherapy $(28.0 \%)$, surgery plus radiochemotherapy $(20.4 \%)$ and other treatments $(9.7 \%)$, including surgery with chemotherapy followed by radiotherapy. The median follow-up period was 62.1 months (range, 1-373 months). The baseline characteristics of the 93 HNSS patients are illustrated in Table I.

Differential analysis between clinicopathological characteristics and outcome statuses. In order to identify the differences between the clinicopathological features of HNSS patients with different outcome statuses, the data of the 93 cases were categorized into three outcome groups, local recurrence, distant metastasis and survival. Each category was further divided into two groups, which resulted in the recurrence, recurrence-free, metastasis, metastasis-free, non-survival and survival groups (Table II). Significant differences in tumor size were identified between the recurrence-free and recurrence, metastasis-free and metastasis, and survival and non-survival groups $(\mathrm{P}=0.001, \mathrm{P}<0.001$ and $\mathrm{P}<0.001$, respectively). In addition, significant differences were found in the pathological differentiation between the recurrence-free and the recurrence $(\mathrm{P}=0.008)$ and survival and non-survival groups $(\mathrm{P}=0.026)$. The logistic regression model was performed to evaluate the risk of recurrence, metastasis and mortality. The risk of tumor recurrence, metastasis and mortality was higher in the patients with a tumor $>5.0 \mathrm{~cm}$ in diameter compared with those with a tumor $\leq 5.0 \mathrm{~cm}$ in diameter (Table III). 


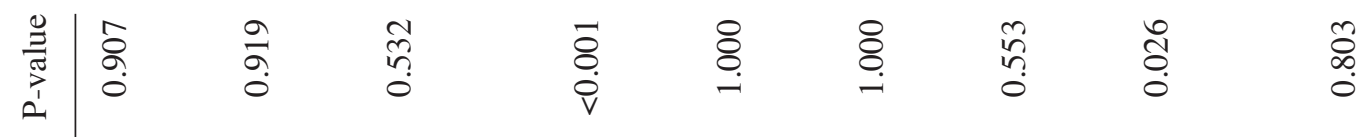

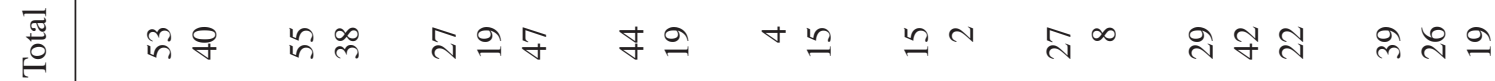

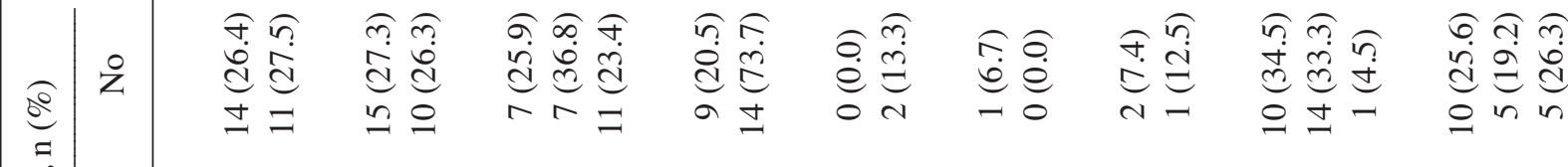

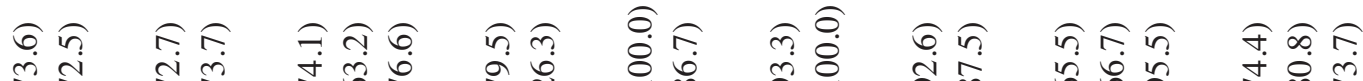

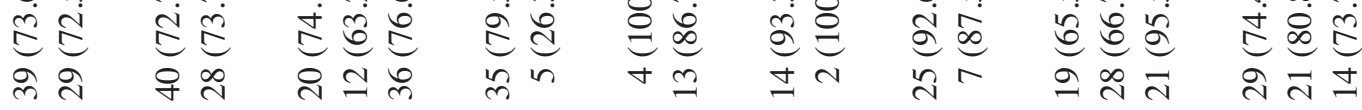

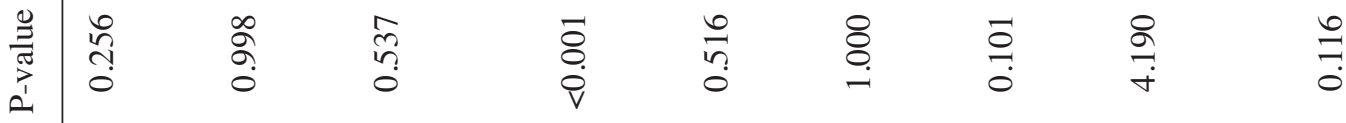

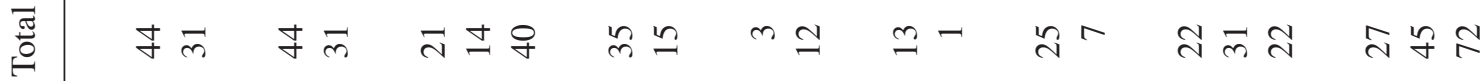

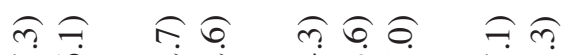

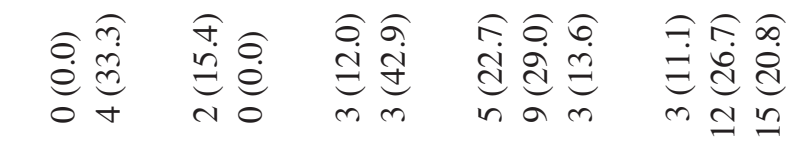
ㄴ i

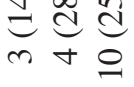

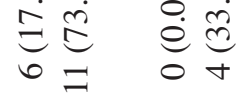

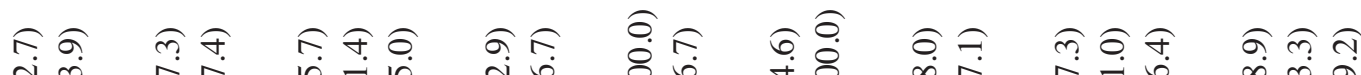

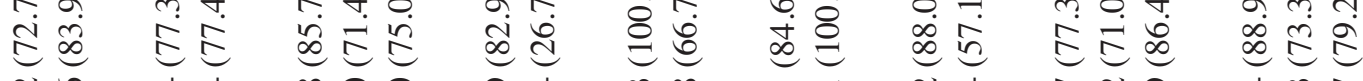

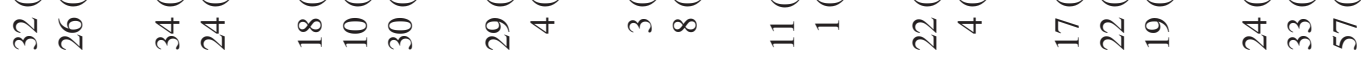

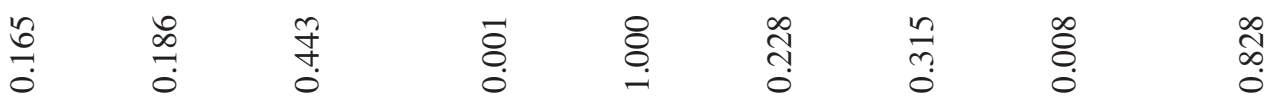

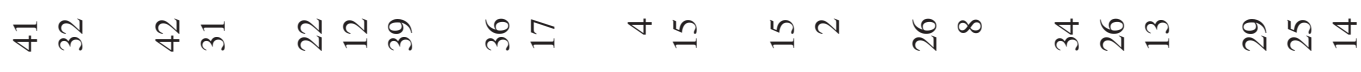

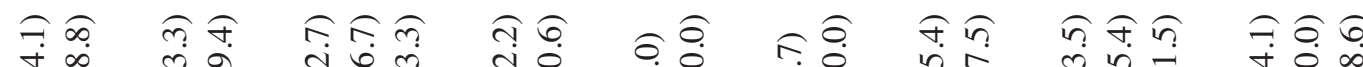
离

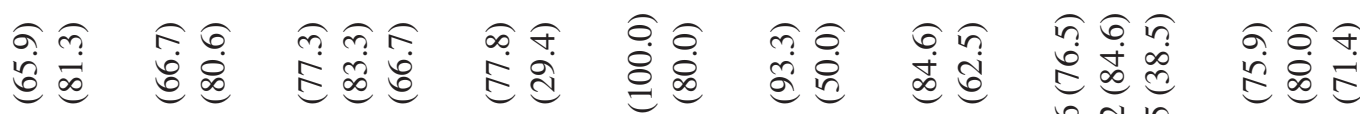
ป⿻一𠃋 ô.

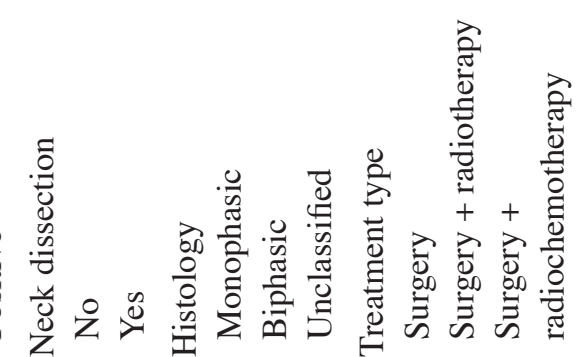


Table III. Logistic regression analysis of risk factors for head and neck synoviosarcoma.

\begin{tabular}{lcc}
\hline Characteristic & Odds ratio $(95 \% \mathrm{CI})$ & P-value \\
\hline $\begin{array}{l}\text { Recurrence } \\
\text { Tumor size }>5.0 \mathrm{~cm}\end{array}$ & $8.400(2.275-31.009)$ & 0.001 \\
$\begin{array}{l}\text { Metastasis } \\
\text { Tumor size }>5.0 \mathrm{~cm}\end{array}$ & $13.292(3.140-56.270)$ & $<0.001$ \\
$\begin{array}{l}\text { Mortality } \\
\text { Tumor size }>5.0 \mathrm{~cm}\end{array}$ & $10.889(3.099-38.261)$ & $<0.001$ \\
\hline CI, confidence interval. & & \\
\hline
\end{tabular}

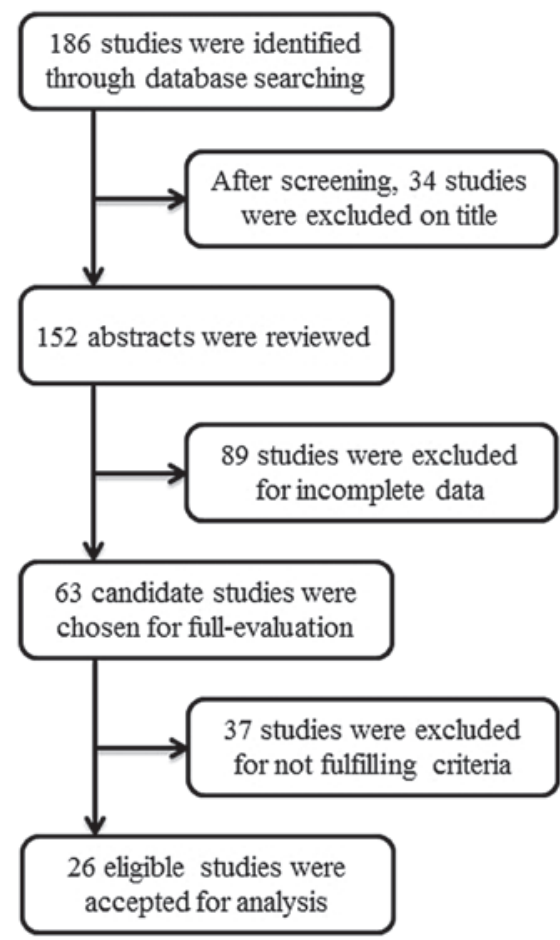

Figure 1. Flow diagram of the process of identifying and selecting studies for the analysis.

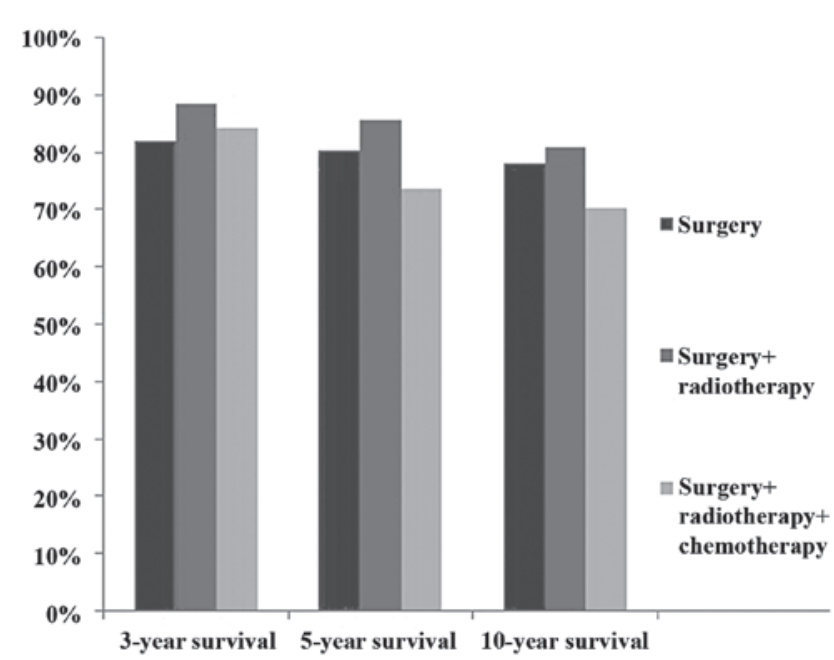

Figure 2. Overall survival rates of head and neck synoviosarcoma patients treated with three different treatment modalities.

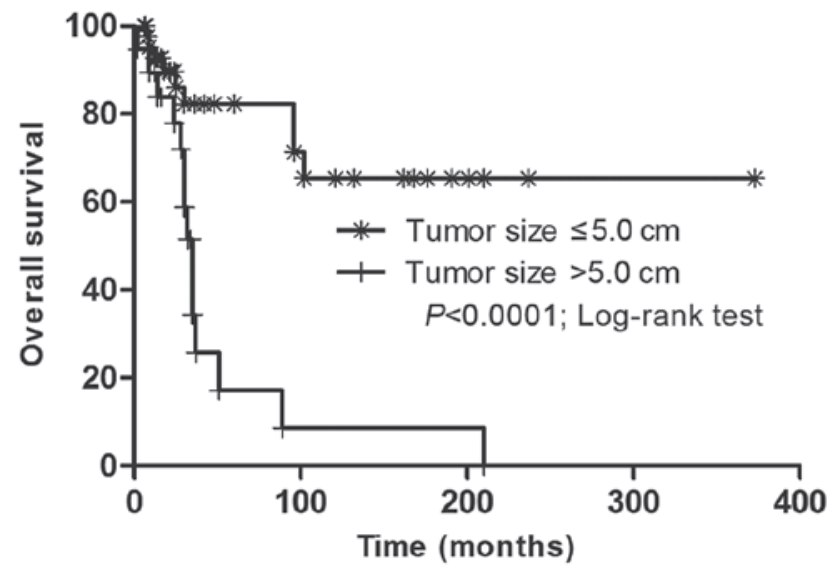

Figure 3. Kaplan-Meier analysis for overall survival of head and neck synoviosarcoma patients depending on tumor size.

Survival and Cox-regression analysis. In total, 20 cases relapsed following the first treatment and the recurrence rate was $21.5 \%$. The distant metastasis and mortality rates were 18.3 and $26.9 \%$, respectively. The three-year survival rate was $82.1 \%$ for surgery alone, $88.5 \%$ for surgery plus radiotherapy and $84.2 \%$ for surgery plus radiochemotherapy. The five-year survival rate was $80.4 \%$ for surgery alone, $85.5 \%$ for surgery plus radiotherapy and $73.7 \%$ for surgery plus radiochemotherapy (Fig. 2). Marked tumor size-dependent differences in the overall survival (OS) rate were revealed (Fig. 3). The Cox proportional-hazards model was utilized to predict the independent prognostic factors for OS. A tumor $>5.0 \mathrm{~cm}$ in diameter was associated with a worse OS rate and the mortality risk increased by 6.460 -fold (95\% CI, 2.206-18.917).

\section{Discussion}

To better elucidate whether the clinicopathological characteristics and treatment were correlated with survival in patients with HNSS and to find specific prognostic factors, a large meta-analysis of 93 patients with histologically confirmed primary HNSS was performed. Surgery is the major treatment for HNSS, resulting in a good prognosis, while surgery-based combined treatment modalities are not statistically superior to surgery alone. In addition, the patients with tumors $>5.0 \mathrm{~cm}$ in diameter have a higher risk of local tumor recurrence, distant metastasis and mortality than those with tumors $\leq 5.0 \mathrm{~cm}$ in diameter. Importantly, the tumor size was the only independent adverse prognostic factor for determining the OS.

Approximately half of the tumors in the 93 cases were located in the upper aerodigestive tract. The upper aerodigestive tract and neck are the most common originating sites of HNSS and they account for $75 \%$ of HNSS. The tumor site determines the clinical presentation of HNSS. Clinically, HNSS is a painless and slow-growing mass, and is usually asymptomatic until it attains a size sufficient to create pressure on the adjacent structures. As a result, those in concealed locations, such as the infratemporal fossa and skull base, which are inaccessible for the clinical examination of a tumor in the early stages, grow unnoticed for a considerable period and the tumors are commonly found at an advanced stage. 
Surgical excision is the mainstay of treatment for HNSS, according to the present study. In total, 93\% of the cases were treated with surgery or surgery plus adjuvant therapy and resulted in a three-year OS rate of $85.3 \%$, five-year OS rate of $81.4 \%$ and 10 -year OS rate of $78.3 \%$. The results in the present study were higher than those previously reported in the former largest analysis with 40 consecutive cases, by the University of Texas MD Anderson Cancer Center (Houston, TX, USA) (9). An explanation for this survival gap is that 19 of 40 cases possessed recurrent disease with positive surgical margins, and a robust association between negative margins and local recurrence-free survival was observed.

The present meta-analysis results are influenced by literature selection biases. However, existing data support the role of adjuvant radiotherapy in improving the local control of HNSS. The patients who received surgery plus radiotherapy achieved good local control and higher survival rates than those treated with surgery alone, although the difference was not statistically significant $(\mathrm{P}=0.19)$. The group of patients who underwent surgery plus radiochemotherapy possessed decreased five- and 10-year OS rates compared with the other two treatment modalities, although it is too soon to conclude that chemotherapy does not improve the OS rate of HNSS since six of the 19 patients in the surgery plus chemoradiotherapy group were diagnosed with advanced-stage disease, either with an extremely large tumor size with extension to adjacent structures, or the patients possessed multiple distant metastasis already. It may be concluded that the early detection of HNSS and total extirpation of the tumor, achieving negative margins, is more effective than employing a salvaging approach at a late stage of tumor development.

Another major interest of the present study was to identify the prognostic factors for HNSS patients. Prognosis in SS has been correlated with the patient age, tumor site, tumor size, mitotic rate, presence of necrosis and histological subtype (35-39). The present study confirms that the tumor size is the only unfavorable prognostic factor for HNSS survival. Certain early studies reported a more favorable outcome in patients with biphasic tumors, whereas other groups found no differences in survival between patients with monophasic tumors and those with biphasic tumors $(36,39,40)$. The present results confirmed the lack of prognostic importance of the histological subtype, even though there were significant differences between the histological subtype and different outcome statuses (Table II).

A few limitations of the present study must be considered. Firstly, even though all the analyzed cases included the treatment outcome and follow-up, certain pieces of important information, including the pathological subtype, surgical margins and tumor extension, were not clearly specified in several cases. Missing these important clinicopathological parameters may influence the results of the present study. Secondly, it is extremely difficult to assemble single center or multicenter prospective trials for an uncommon disease such as HNSS. Thus, the retrospective data makes selection bias a possibility.

Despite its limitations, the present meta-analysis comprehensively analyzed the clinicopathological features of HNSS from the sporadic case reports in the peer-reviewed English literature to date. Surgical excision is a mainstream treatment of HNSS. Post-operative adjuvant radiotherapy is effective in local tumor control and improves the OS rate of HNSS. However, the effectiveness remains to be validated in further multicenter, longitudinal, prospective, large cohort studies. In addition, the present study confirmed that a tumor size $>5.0 \mathrm{~cm}$ in diameter was an independent adverse prognostic factor for OS.

\section{Acknowledgements}

This study was supported by the Shanghai Committee of Science and Technology (grant no. 12DZ2260100).

\section{References}

1. Gurney JG, Young JL Jr, Roffers SD, et al: Soft tissue sarcomas. In: Cancer Incidence and Survival Among Children and Adolescents: United States SEER Program 1975-1995. Gloeckler Ries LA, Smith MA, Gurney JG, Linet M, Tamra T, Young JL Jr, Bunin GR (eds). National Cancer Institute, SEER program, Bethesda, MA, USA, pp111-123, 1999.

2. Bergh P, Meis-Kindblom JM, Gherlinzoni F, et al: Synovial sarcoma: identification of low and high risk groups. Cancer 85: 2596-2607, 1999.

3. Ichinose H, Wickstrom JK, Hoerner HE and Derbes VL: The early clinical presentation of synovial sarcoma. Clin Orthop Relat Res 185-189, 1979.

4. Spillane AJ, A'Hern R, Judson IR, Fisher C and Thomas JM: Synovial sarcoma: a clinicopathologic, staging, and prognostic assessment. J Clin Oncol 18: 3794-3803, 2000.

5. Kawai A, Woodruff J, Healey JH, et al: SYT-SSX gene fusion as a determinant of morphology and prognosis in synovial sarcoma. N Engl J Med 338: 153-160, 1998.

6. Åkerman M, Ryd W and Skytting B: Fine-needle aspiration of synovial sarcoma: Criteria for diagnosis: Retrospective reexamination of 37 cases, including ancillary diagnostics. A Scandinavian sarcoma group study. Diagn Cytopathol 28: 232-238, 2003.

7. Cormier JN and Pollock RE: Soft tissue sarcomas. CA Cancer J Clin 54: 94-109, 2004.

8. Al-Daraji W, Lasota J, Foss R and Miettinen M: Synovial sarcoma involving the head: nalysis of 36 cases with predilection to the parotid and temporal regions. Am J Surg Pathol 33: 1494-1503, 2009.

9. Harb WJ, Luna MA, Patel SR, et al: Survival in patients with synovial sarcoma of the head and neck: association with tumor location, size, and extension. Head Neck 29: 731-740, 2007.

10. Barkan GA and El-Naggar AK: Primary synovial sarcoma of the parotid gland. Ann Diagn Pathol 8: 233-236, 2004.

11. Fisher RM and Spiro PC: Cervical synovial sarcoma in a young boy. S Afr Med J 48: 2181-2182, 1974.

12. Saydam L, Kizilay A, Kalcioglu MT, Mizrak B and Bulut F: Synovial sarcoma of the pharynx: a case report. Ear Nose Throat J 81: 36-39, 2002.

13. Bertolini F, Bianchi B, Pizzigallo A, Tullio A and Sesenna E: Synovial cell sarcoma of the neck. Case report and review of the literature. Acta Otorhinolaryngol Ital 23: 391-395, 2003.

14. Agada FO, Murphy J, Sharma R, Karsai L and Stafford ND: Biphasic synovial sarcoma of the posterior pharyngeal wall: a case report. Ear Nose Throat J 84: 302, 304, 306, 2005.

15. Yildirim A, Tosun F and Alaomeroglu M: Synovial sarcoma of the nasal septum. Ann Otol Rhinol Laryngol 114: 84-86, 2005.

16. Bukawa H, Kawabata A, Murano A, et al: Monophasic epithelial synovial sarcoma arising in the temporomandibular joint. Int J Oral Maxillofac Surg 36: 762-765, 2007.

17. Orlandi E, Zonca G, Pignoli E, et al: Postoperative radiotherapy for synovial sarcoma of the head and neck during pregnancy: clinical and technical management and fetal dose estimates. Tumori 93: 45-52, 2007.

18. Lai V, Farrag TY, Cao D, et al: Synovial sarcoma of the infratemporal fossa. Am J Otolaryngol 28: 444-447, 2007.

19. Luo CW, Liu CJ and Chang KM: Synovial sarcoma of the temporomandibular joint area: report of a case. Oral Surg Oral Med Oral Pathol Oral Radiol Endod 104: e62-e65, 2007.

20. Jay A, Hutchison I, Piper K, Farthing PM and Richards PS: Synovial sarcoma presenting as a parotid mass: case report and review of literature. Head Neck 30: 1654-1659, 2008.

21. Ishiki H, Miyajima C, Nakao K, Asakage T, Sugasawa M and Motoi T: Synovial sarcoma of the head and neck: rare case of cervical metastasis. Head Neck 31: 131-135, 2009. 
22. Blankenburg S, Petersen I, Katenkamp D and Chilla R: An unusual case of a synovial sarcoma of the parotid gland in an elderly patient. Auris Nasus Larynx 38: 523-527, 2011.

23. Sato T, Hasegawa H, Sugasawa M, et al: Free jejunal transfer for a 15 -year-old girl with synovial sarcoma of the hypopharynx. J Plast Reconstr Aesthet Surg 64: 1100-1103, 2011.

24. Rigante M, Visocchi M, Petrone G, Mulè A and Bussu F: Synovial sarcoma of the parotid gland: a case report and review of the literature. Acta Otorhinolaryngol Ital 31: 43-46, 2011.

25. Dhawan A, Shenoy AM, Chavan P, Sandhu S and Sriprakash D: Synovial sarcoma of the infratemporal fossa with extension into the oral cavity - a rare presentation and literature review. J Oral Maxillofac Surg 70: 2923-2929, 2012.

26. Khademi B, Mohammadianpanah M, Ashraf MJ and Yeganeh F: Synovial sarcoma of the parapharyngeal space. Auris Nasus Larynx 34: 125-129, 2007.

27. Bilgic B, Mete O, Oztürk SA, Demiryont M, Keles N and Basaran M: Synovial sarcoma: a rare tumor of larynx. Pathol Oncol Res 9: 242-245, 2003.

28. Tamarit Conejeros JM, Estrems Navas P, Estellés Ferriol E and Dalmau Galofre J: Synovial sarcoma of the infratemporal fossa. Acta Otorrinolaringol (English Edition) 61: 389-391, 2010.

29. Kikuchi I, Anbo J, Nakamura S, et al: Synovial sarcoma of the thyroid. Report of a case with aspiration cytology findings and gene analysis. Acta Cytol 47: 495-500, 2003

30. Kartha SS and Bumpous JM: Synovial cell sarcoma: diagnosis, treatment, and outcomes. Laryngoscope 112: 1979-1982, 2002.

31. Wang H, Zhang J, He X and Niu Y: Synovial sarcoma in the oral and maxillofacial region: report of 4 cases and review of the literature. J Oral Maxillofac Surg 66: 161-167, 2008.
32. Capelli M, Bertino G, Morbini P, Proh M, Falco CE and Benazzo M: CO2 laser in the treatment of laryngeal synovial sarcoma: a clinical case. Tumori 93: 296-299, 2007.

33. Meer S, Coleman $\mathrm{H}$ and Altini M: Oral synovial sarcoma: a report of 2 cases and a review of the literature. Oral Surg Oral Med Oral Pathol Oral Radiol Endod 96: 306-315, 2003.

34. Alberty J and Dockhorn-Dworniczak B: Monophasic synovial sarcoma of the neck in an 8-year-old girl resembling a thyroglossal duct cyst. Int J Pediatr Otorhinolaryngol 63: 61-65, 2002.

35. Roth JA, Enzinger FM and Tannenbaum M: Synovial sarcoma of the neck: a followup study of 24 cases. Cancer 35: 1243-1253, 1975.

36. Cagle LA, Mirra JM, Storm FK, Roe DJ and Eilber FR: Histologic features relating to prognosis in synovial sarcoma. Cancer 59: 1810-1814, 1987.

37. Rööser B, Willén H, Hugoson A and Rydholm A: Prognostic factors in synovial sarcoma. Cancer 63: 2182-2185, 1989.

38. Brodsky JT, Burt ME, Hajdu SI, Casper ES and Brennan MF Tendosynovial sarcoma. Clinicopathologic features, treatment, and prognosis. Cancer 70: 484-489, 1992.

39. Singer S, Baldini EH, Demetri GD, Fletcher JA and Corson JM: Synovial sarcoma: prognostic significance of tumor size, margin of resection, and mitotic activity for survival. J Clin Oncol 14: 1201-1208, 1996.

40. Krall RA, Kostianovsky M and Patchefsky AS: Synovial sarcoma: a clinical, pathological, and ultrastructural study of 26 cases supporting the recognition of a monophasic variant. Am J Surg Pathol 5: 137-151, 1981. 